\title{
Mouse Hepatitis Virus Infection, Intestine, Mouse
}

\author{
Stephen W. Barthold
}

Synonyms. Enterotropic coronavirus infection, mouse, lethal intestinal virus of infant mice (LIVIM)

\section{Gross Appearance}

Enterotropic mouse hepatitis virus (MHV) infection is usually subclinical, with no gross lesions. Neonatal mice suffer high mortality when the virus is first introduced to a naive breeding population. They become dehydrated, with soiling of the perineum with yellow, pasty feces. Their stomachs are usually empty, and intestines are thin-walled, flaccid, and contain watery yellow digesta and gas. Juvenile mice are less severely affected, but are often runted with pot bellies and oily-appearing hair. Careful examination of weaning-age or adult mice may reveal dark, sticky feces and opaque, thickened segments of bowel. Livers, if affected, have few to many small, pale or hemorrhagic foci (see "Mouse Hepatic Virus Infection, Liver, Mouse," this volume; Barthold 1986; Barthold and Smith 1984; Barthold et al. 1982, 1993; Broderson et al. 1976; Hierholzer et al. 1979; Ishida and Fujiwara 1979; Ishida et al. 1978; Kraft 1962, 1966). Athymic nude mice infected with enterotropic MHV may have segmental thickening of the cecum and ascending colon, with enlargement of mesenteric lymph nodes (Barthold et al. 1985; Ward et al. 1977).

\section{Microscopic Features}

The quality and location of intestinal lesions vary widely, depending on MHV strain and host age. Enterotropic MHV characteristically causes mucosal epithelial necrosis and syncytium formation, while host responses include inflammation and compensatory mucosal hyperplasia. In neonatal mice, viral effects are severe. Syncytia can be pronounced in the small intestine, since they tend to be retained at villus tips or are detached within the lumen. These large, multinucleate cells have been termed "balloon cells" because of their appearance (Fig. 343). Infected enterocytes can have poorly defined eosinophilic intracytoplasmic inclusions, but these are of little diagnostic value. When cytolysis predominates, villi become markedly attenuated (Fig. 344). Syncytia tend to form as large masses in the surface mucosa of the large bowel (Fig. 345), but the mucosal epithelium is often nearly completely effaced (Fig. 346). The ascending colon seems to be a preferential target for enterotropic MHV, regardless of host age or strain. Lesions are most apt to be present in these segments of bowel (Barthold et al. 1993). Surviving mice or weaningage mice respond to infection with transient mucosal hyperplasia of the involved segment of bowel (Fig. 347). Lesions in adult mice are marginal, consisting of only a few scattered syncytia and modest crypt hyperplasia (Barthold 1986; Barthold et al. 1982, 1993; Biggers et al. 1964; Hierholzer et al. 1979; Ishida and Fujiwara 1979; Ishida et al. 1978; Kraft 1962, 1966). Athymic nude mice can develop chronic mucosal hyperplasia with syncytia, erosion, and inflammation (Barthold et al. 1985; Ward et al. 1977). Alternatively, nude mice infected as adults or with avirulent strains of virus may have minimal enteric lesions, with only a few syncytia present in surface mucosa and minimal hyperplasia or inflammation.

\section{Ultrastructure}

Infected enterocytes often have nonspecific degenerative changes or are undergoing necrosis. The endoplasmic reticulum contains 80 - to $120-\mathrm{nm}$ 


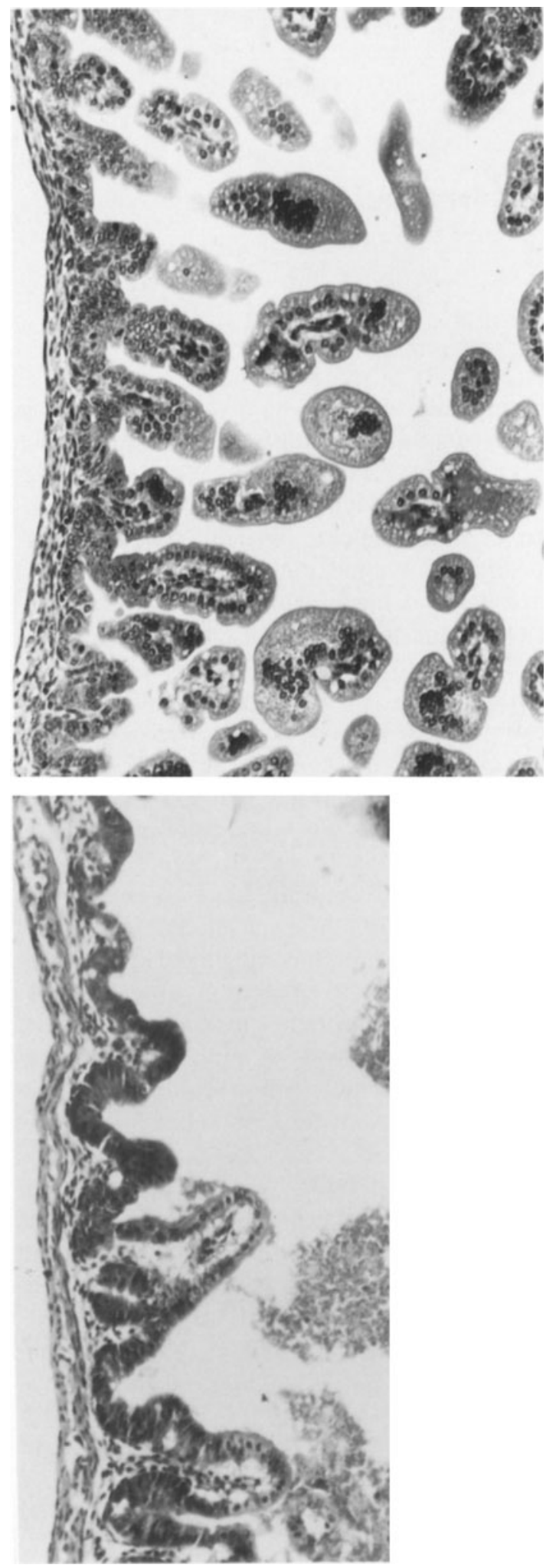

coronaviral particles, and the cytoplasm may possess one or more amorphous electron-dense bodies and complexes of reticular structures (Fig. 348). These changes are seen in other infected tissues as well (see "Mouse Hepatic Virus Infection, Liver, Mouse," this volume). Virus particles can also be found within macrophages of the lamina propria.

Ultrastructural correlates of inclusions seen by light microscopy have not been found (Barthold et al. 1982; Hierholzer et al. 1979; Ishida and Fujiwara 1979; Ishida et al. 1978).

\section{Differential Diagnosis}

Enteric disease in mice can be caused by a number of viruses, bacteria, and protozoa, but histologic changes, particularly epithelial syncytia in mice infected with MHV, are diagnostic. Because lesions are often segmental, all levels of bowel should be examined, but lesions are most apt to be present in the ascending colon and cecum. The most sensitive means of detecting MHV exposure in mice is to perform serologic tests for antibody, as most infections are subclinical with minimal microscopic lesions.

\section{Biologic Features}

\section{Natural History}

Mouse hepatitis virus is a highly mutable coronavirus with many, constantly evolving and changing strains. Host immunity to MHV is virus strain specific, and therefore mice can be repeatedly infected with newly mutated or introduced virus strains (Barthold 1986; Barthold and Smith 1989; Homberger et al. 1992). Enterotropic strains of the virus are highly contagious. When first introduced to a breeding population of mice, epizootics of enteritis with diarrhea can be associated with high morbidity and mortality among in-

Fig. 343. (above) Small intestine, neonatal mouse infected with enterotropic mouse hepatic virus (MHV). Villi possess several large multinucleate syncytia ("balloon cells") which are typical for MHV. H\&E, $\times 150$

Fig. 344. (below) Small intestine, neonatal mouse infected with enterotropic MHV. Villi are markedly attenuated and crypts are hypercellular. (Courtesy of Dr. S. W. Barthold and Hemisphere Publishing Corp.) H\&E, $\times 150$ 

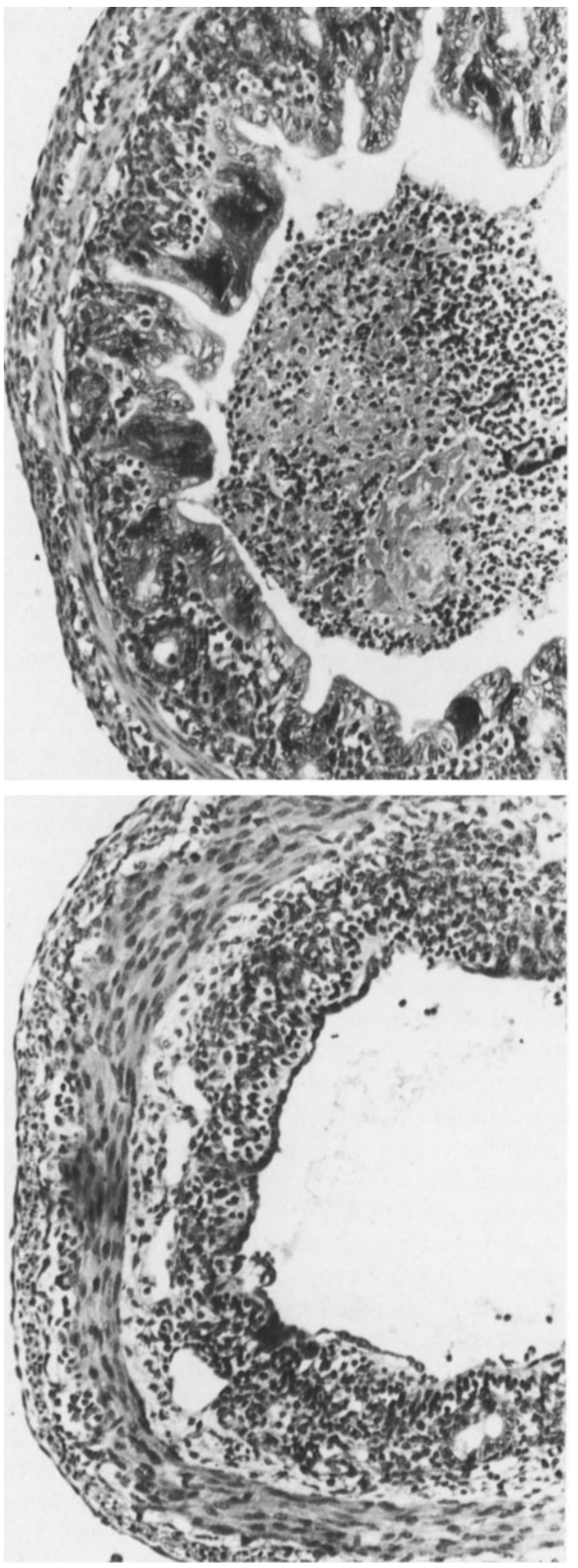

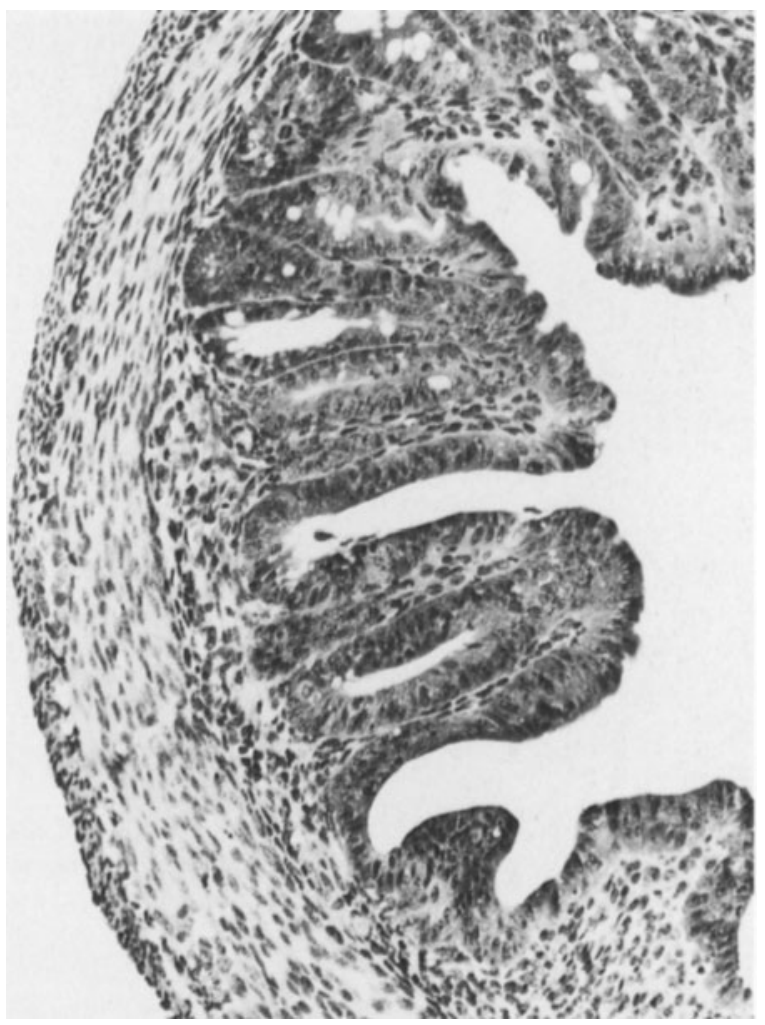

Fig. 345. (upper left) Colon neonatal mouse infected with enterotropic MHV. The lumen contains leukocytes and cellular debris. The surface mucosa has multiple prominent epithelial syncytia. H\&E, $\times 150$

Fig. 346. (below) Cecum, neonatal mouse infected with enterotropic MHV. The mucosal epithelium has been almost completely effaced, leaving only the lamina propria, except for an incomplete layer of surface epithelium. $H \& E, \times 150$

Fig. 347. (upper right) Cecum, neonatal mouse recovering from enterotropic MHV infection. The mucosa is distorted due to hyperplasia of the crypt epithelium. H\&E, $\times 150$ 


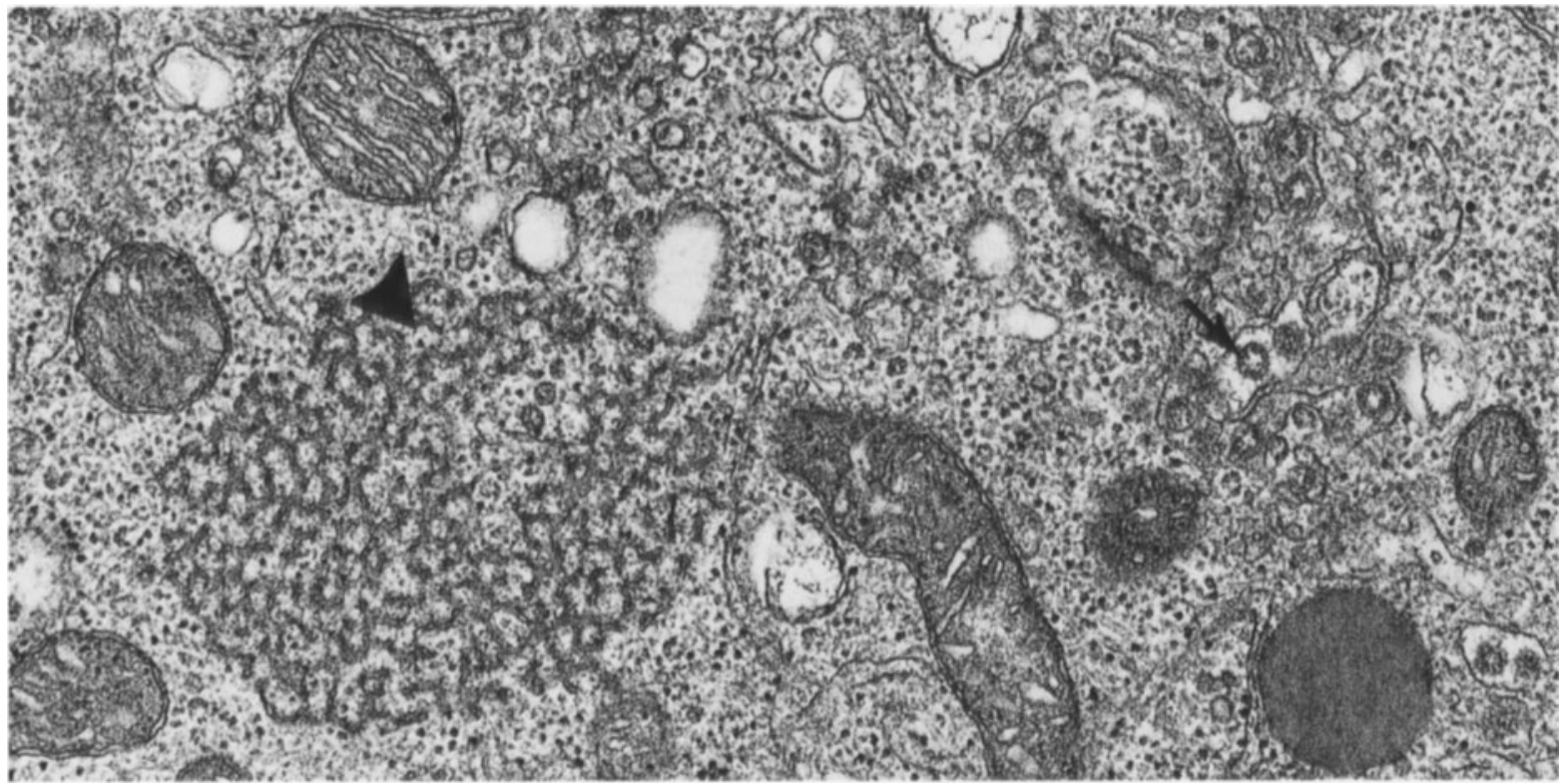

Fig. 348. Electron micrograph of cecal mucosal epithelium of a neonatal mouse infected with MHV. The cytoplasm contains an electron-dense reticular structure. Virus particles are present in cisternae of the endoplasmic reticulum. (Courtesy of Dr. S. W. Barthold and Laboratory Animal Science) Uranyl acetate and lead citrate, $\times 225000$ fant mice. Once enzootic within a population, signs of disease are less obvious, since pups from recovered dams are protected by maternal antibody during the neonatal period (Barthold et al. 1988; Homberger 1992; Homberger and Barthold 1992; Hierholzer et al. 1979; Ishida and Fujiwara 1982; Ishida et al. 1978). Under these circumstances, reduced litter survival and runting occur, but may not be noticed. Mice of all ages and genotypes are susceptible to infection, but infection among adults is usually subclinical. With the exception of athymic nude or other immunodeficient mice which develop persistent infections, mice normally recover within 2 weeks of infection (Barthold 1986; Barthold and Smith 1984; Barthold et al. 1982, 1993; Biggers et al. 1964).

\section{Pathogenesis}

Respiratory strains of MHV initially replicate in nasal epithelium and then disseminate to multiple organs. These viruses have been extensively studied and are known to be influenced by host genotype, age, and lymphoreticular function. These viral strains have little or no tropism for bowel (see "Mouse Hepatic Virus Infection, Liver, Mouse," this volume). Enterotropic strains cause infections largely limited to bowel, although limited dissemination to other organs may take place (Barthold and Smith 1984; Barthold et al. 1993; Hierholzer et al. 1979; Ishida and Fujiwara 1979; Ishida et al. 1978). Neonatal mice develop severe enteritis, resulting in malabsorption and diarrhea. As mice mature, intestinal cell turnover rate is accelerated, allowing a compensatory proliferative response to viral damage (Barthold et al. 1993; Biggers et al. 1964). Genotype and maturation of immune response no doubt also influence infection, but age is the most critical determinant of host susceptibility to enterotropic MHVinduced disease. Mice of all ages are susceptible to infection and support nearly equal levels of virus replication, whether or not disease is apparent (Barthold et al. 1993). Colostral immunoglobulin (Ig)A and IgG antibody from immune mothers is protective against fatal infections in neonatal mice (Homberger 1992; Homberger and Barthold 1992; Ishida and Fujiwara 1982). Athymic nude mice develop persistent enteric infections, with chronic mucosal hyperplasia (Barthold et al. 1985). The distribution of lesions within the intestine is probably determined by virus strain. Severe small intestine involvement has been noted with some strains, such as LIVIM, MHV-S/CDC, and MHVD (Biggers et al. 1964; Hierholzer et al. 1979; 
Ishida and Fujiwara 1979; Ishida et al. 1978). Predilection for the large bowel has been noted with other MHV isolates, such as MHV-Y (Barthold et al. 1982, 1993; Barthold and Smith 1984). Intestinal bacteria and protozoa may influence the course of enteric MHV infections, but little work has been done on this aspect. Recovered mice are immune to reinfection with the homologous MHV strain, but can be reinfected with an antigenically heterologous strain (Barthold and Smith 1989; Barthold et al. 1993). Because of the high degree of tissue selectivity, enterotropic strains tend not to disseminate to other tissues and are therefore not likely to be transmitted in utero (Barthold et al. 1988).

\section{Etiology}

Many strains of this virus exist which can be partially differentiated antigenically, but all share common antigens with each other and with coronaviruses of rats and humans (Barthold 1986). Antigenic composition does not predict virulence or organotropism (Barthold and Smith 1984).

\section{Frequency}

As discussed in "Mouse Hepatic Virus Infection, Liver, Mouse" (this volume), MHV is among the most common viruses of laboratory mice. Enterotropic strains seem to be more prevalent than respiratory strains, but this may be influenced by the relative ease of diagnosing enterotropic infections. With thorough analysis, enteric lesions are consistently found in actively infected mice, but are detected most easily in young mice. Lesions are present for only 1-2 weeks and are absent when seroconversion takes place, unless immune mice have been reinfected with a new strain of the virus.

\section{Comparison with Other Species}

Enterotropic MHV infections are analogous to enteric coronavirus infections in many other species of birds and mammals. Enteric coronaviruses have been described in humans, nonhuman primates, turkeys, swine, cattle, sheep, horses, dogs, rats, cats, and rabbits. As in mice, these viruses are generally associated with disease in neonates. Small intestine changes in neonates usually include villus "atrophy," resulting in malabsorption and diarrhea (Barthold 1986).

\section{References}

Barthold SW (1986) Mouse hepatitis virus biology and epizootiology. In: Bhatt PN, Jacoby RO, Morse HC III, New AE (eds) Viral and mycoplasmal infections of laboratory rodents: effects on biomedical research, chap 25. Academic, Orlando, pp 571-601

Barthold SW, Smith AL (1984) Mouse hepatitis virus s\&mrelated patterns of tissue tropism in suckling mice. Arch Virol 81:103-112

Barthold SW, Smith AL (1989) Virus strain specificity of challenge immunity to coronavirus. Arch Virol 104:187-196

Barthold SW, Smith AL, Lord PF, Bhatt PN, Jacoby RO, Main AL (1982) Epizootic coronaviral typhlocolitis in suckling mice. Lab Anim Sci 32:376-383

Barthold SW, Smith AL, Povar ML (1985) Enterotropic mouse hepatitis virus infection in nude mice. Lab Anim Sci 35:613618

Barthold SW, Beck DS, Smith AL (1988) Mouse hepatitis virus and host determinants of vertical transmission and maternally-derived passive immunity in mice. Arch Virol 100:171183

Barathold SW, Beck DS, Smith AL (1993) Enterotropic coronavirus (mouse hepatitis virus) in mice: influence of host age and strain on infection and disease. Lab Anim Sci 43:276284

Biggers DC, Kraft LM, Sprinz H (1964) Lethal intestinal virus infection of mice (LIVIM). An important new model for study of the response of the intestinal mucosa to injury. Am J Pathol 45:413-422

Broderson JR, Murphy FA, Hierholzer JC (1976) Lethal enteritis in infant mice caused by mouse hepatitis virus. Lab Anim Sci 26:824

Hierholzer JC, Broderson JR, Murphy FA (1979) New strain of mouse hepatitis virus as the cause of lethal enteritis in infant mice. Infect Immun 24:508-522

Homberger FR (1992) Maternally-derived passive immunity to enterotropic mouse hepatitis virus. Arch Virol 122:133141

Homberger FR, Barthold SW (1992) Passively acquired challenge immunity to enterotropic coronavirus in mice. Arch Virol 126:35-43

Homberger FR, Barthold SW, Smith AL (1992) Duration and strain specificity of immunity to enterotropic mouse hepatitis virus. Lab Anim Sci 42:347-351

Ishida T, Fujiwara K (1979) Pathology of diarrhea due to mouse hepatitis virus in the infant mouse. Jpn J Exp Med 49:33-41

Ishida T, Fujiwara K (1982) Maternally derived immune resistance to fatal diarrhea in infant mice due to mouse hepatitis virus. Jpn J Exp Med 52:231-235

Ishida T, Taguchi F, Lee Y-S, Yamada A, Tamura T, Fujiwara $\mathrm{K}$ (1978) Isolation of mouse hepatitis virus from infant mice with fatal diarrhea. Lab Anim Sci 28:269-276

Kraft LM (1962) An apparently new lethal virus disease of infant mice. Science 137:282-283 
Kraft LM (1966) Epizootic diarrhea of infant mice and lethal intestinal virus infection of infant mice. Natl Cancer Inst Monogr 20:55-61
Ward JM, Collins MJ, Parker JC (1977) Naturally occurring mouse hepatitis virus infection in the nude mouse. Lab Anim Sci 27:372-376

\title{
Murine Rotavirus Infection, Intestine, Mouse
}

\author{
Stephen W. Barthold
}

Synonyms. Epidemic diarrhea of infant mice, epizootic diarrhea of infant mice (EDIM)

\section{Gross Appearance}

Mice with murine rotavirus infection less than 18 days of age have mustard-colored to dark sticky feces which adhere to the perineum and give an oily appearance to the hair. Rarely, dried feces obstruct the anus, resulting in obstipation. Affected mice are pot-bellied and runted, but recover their weight by 8 weeks of age. The stomach is full of ingesta, and the intestines are distended with copious mucoid yellow digesta and gas. Older mice do not manifest signs or lesions (Cheever 1956; Cheever and Mueller 1947; Kraft 1957, 1958, 1962; Sheridan et al. 1983).

\section{Microscopic Features}

Light microscopic lesions are difficult to recognize and are often absent, particularly in older mice. Tips of small intestinal villi, especially in the jejunum and ileum, appear bulbous due to swelling of epithelial cells, vascular congestion, and dilatation of lymphatics (Fig. 349). Columnar epithelial cytoplasm is swollen due to diffuse, fine vesiculation. Severely affected cells develop large cytoplasmic vacuoles, and $1-$ to $4-\mu \mathrm{m}$ acidophilic inclusions, which are not diagnostic, may be randomly distributed within the cytoplasm. Minimal or no inflammation is present (Adams and Kraft 1967; Kraft 1957, 1962; Pappenheimer and Cheever 1948).

\section{Ultrastructure}

Enterocytes infected with the virus contain numerous vesicles arising from rough endoplasmic reticulum. These vesicles contain many virus particles, electron-dense granular material, and lipid. The cytoplasm also has aggregates of dense granular material with nascent virus particles that range from 65 to $80 \mathrm{~nm}$ in diameter (Fig. 350). The largest particles possess a double set of membranes; the outer membrane is acquired by budding into the lumen of the endoplasmic reticulum. Smaller particles with only a single membrane are also present in vesicles and in the cytoplasmic matrix. Tubular structures with single or double sets of membranes may be present in the cytoplasm, endoplasmic reticulum, and, occasionally, the nucleus. These structures apparently represent products of abnormal virus assembly (Fig. 351). No structures corresponding to the intracytoplasmic inclusions seen by light microscopy can be found ultrastructurally. Virus is present in enterocytes at all levels of the small and large intestine. In the small intestine, there is a gradient of cellular susceptibility toward villus tips. Virus is released into the intestinal lumen by cell disruption and exfoliation (Adams and Kraft 1963, 1967; Banfield et al. 1968; Holmes et al. 1975; Kraft 1962; Osborne et al. 1988; Starkey et al. 1986).

\section{Differential Diagnosis}

Agents which produce diarrhea in young mice include enterotropic mouse hepatitis virus (MHV), reovirus, Salmonella, and Spironucleus muris. Coinfection among these agents is frequent. Murine rotavirus infection is difficult to diagnose, since morphological lesions are often subtle or absent even in the presence of clinical disease. Superb histotechnique is required for appropriate examination, since autolysis and mishandling of tissues are most apt to affect mucosa at the villus tips. Neonatal enterocytes possess apical tubular systems involved in uptake of colostrum, giving 